\title{
Renal Cell Carcinoma - How Can We Predict its Outcomes in Clinical Practice?
}

Ieva Vaivode, Vilnis Lietuvietis, Alinta Hegmane, Iveta Kudaba

Riga East University Hospital, Latvian Oncology Centre, Riga, Latvia

\begin{abstract}
Summary
Morbidity and mortality data of RCC (renal cell carcinoma) differs a lot among the European countries. In Latvia a growing trend in both incidence and mortality rates is still observed. The expanding availability of multiple treatment strategies has increased the importance of skilled individualized outcome prediction for patients. Several prognostic factors are available in RCC including anatomical, histological, clinical and molecular ones, but none of them is very precise, when used alone. Therefore increasing number of prognostic systems has been created in local and metastatic disease to increase predictive accuracy. In order to encourage the clinicians to use the available models in their routine practice, we tried to select the most relevant ones and include them in a simple algorithm to be used in common clinical scenarios throughout entire history of the disease in patients with RCC.
\end{abstract}

Key words: renal cell carcinoma; prognostic factors; prognostic models.

\section{INTRODUCTION}

RCC accounts for approximately $90 \%$ of all kidney malignancies and represents $2-3 \%$ of all cancers, with the highest incidence in Western countries (28). During the last two decades an annual increase of about $2 \%$ in incidence is observed both worldwide and in Europe, however in some countries, as Denmark and Sweden decrease is reported. In Europe, overall mortality rates for RCC have increased until the early 1990s, but thereafter the situation has very much changed in some European countries and less in others. Decrease in mortality rates is observed in Scandinavian countries already since the 1980s, but in Austria and Netherlands, since the early 1990s. However, in some European countries, including Latvia, mortality rates still show an upward trend (10). Management of metastatic RCC has significantly changed in the last few years with the development of targeted therapies. At present, several antiangiogenic drugs: tyrosine kinase inhibitors, monoclonal antibodies have been approved both in the USA and in Europe for the treatment of metastatic RCC. Significant differences in mortality rates among the European countries could be explained by the limited availability of the new treatments. At present, in Latvia none of the new medications is completely reimbursed and just a small number of patients have been on the targeted treatment within investigational protocols or individual compensation request. Taking into consideration the growing treatment costs and limited efficacy, that has to be balanced with the risk of side effects, physicians involved in the treatment of RCC, especially in the countries like Latvia with limited financial resources, increasingly need accurate tools in prediction of treatment response in order to select the patients who may benefit most.
RCC - DIFFICULT TO PREDICT AND TO MANAGE

RCC is a heterogeneous and complex disease with variable prognosis. Treatment decision and creation of appropriate follow-up plan should be based on prediction of response to therapy and on the prognosis of recurrence and survival. Unfortunately in case of RCC there is no specific marker to help in monitoring the disease and estimating treatment efficacy. Several non-specific factors can be used to predict the disease outcomes, however none of them is perfectly accurate when used alone. Therefore a number of prognostic models in the form of scoring algorithms or nomograms combining different prognostic variables have been developed in order to improve predictive accuracy.

At present TNM stage is the most important prognostic tool in RCC, and most likely will remain so in the future. Patients with stage pT1 or pT2 (organ confined) disease have the best prognosis, with 5-year cancerspecific survival rate after nephrectomy ranging from $67 \%$ to $94 \%$; for patients with locally advanced tumors it decreases by $23 \%$ to $67 \%$, and once RCC has metastasized, it is less than $23 \%$ (45). However in real life individual patient cases show significant differences in tumor behavior within all stages, resulting in variable survival prognosis.

Research continues to detect strong and easily available prognostic parameters that may help to classify patients into groups with different risks for death from renal cancer. As a result in the last 15 years a number of prognostic systems combining several independent factors have been developed to improve the predictive accuracy provided just by TNM stage. An important advantage of these models, based on mathematical calculations and statistical estimates, is their ability to measure the predictive accuracy, which enables all new variables to be objectively evaluated. In the world of extremely rapid flow of information, we tried to compile 
the data published so far, by selecting the most important prognostic factors and their integrated systems in order to make them more applicable in everyday clinical use. A literature review was performed using the PubMed database for articles published by January 1, 2013. As the first step we examined the systematic reviews and meta-analysis, followed by the analyses of each study data.

Classical prognostic factors for RCC can be divided into anatomical, histological, clinical and molecular, which differ in local and metastatic disease (43). We tried to identify the ones that can be easily detected in routine practice by the clinicians.

\section{PROGNOSTIC FACTORS IN NON-METASTATIC RCC}

Anatomical factors are the ones, that are integrated in the TNM staging system (tumor node metastasis) and include: tumor size, growth beyond renal capsule into fat or peri-sinus tissues, invasion of renal vein and/or inferior vena cava, adrenal extension and lymph node invasion. The TNM classification undergoes continuous improvements, each version, including the last 2009 has introduced significant changes based on recent studies, because of their prognostic relevance (28). As an example: in previous TNM classifications, the pT3b group included both renal vein and inferior vena cava invasions, that have been separated in the latest version of the TNM classification; adrenal invasion has a poor prognosis, so it has been re-classified as pT4 tumor.

There are still some unresolved issues regarding TNM staging. One of them is related to nodal invasion, which has been confirmed to be an independent prognostic factor regardless of $\mathrm{T}$ stage. However, the necessity of the sub-classification of nodal involvement according to the number of affected nodes ( $\mathrm{Nl}$ : l node involved; N2: > 1 node involved) is not clear. Furthermore, in the 2002 TNM classification it is recommended that at least 8 nodes should be removed for nodal staging. Below this number of nodes, the patient should be staged as Nx. In clinical practice, this number of removed nodes is not always achieved, especially in case of partial or laparoscopic nephrectomy $(28,42,43)$.

Another issue applies to renal sinus fat invasion, which has been classified as pT3a since the 2002 version of the TNM classification. However, data suggest that renal sinus fat invasion carries a worse prognosis than perinephric fat invasion and therefore should not be included in the same stage group (1).

Histological factors. According to the World Health Organization (WHO) there are 3 major histological subtypes of RCC: clear cell, papillary and homophobe (28). At least few studies are proving significant correlation between histologic subtype and disease specific survival in univariate analysis, with clear cell RCC being the most aggressive tumor followed by papillary and homophobe. Papillary tumors are divided into two groups with very different prognosis: type I and II. However, the prognostic value reduces in multivariate analysis, suggesting that stage and grade have a higher impact on prognosis than histologic subtype. Histological features of the highest prognostic value include: Fuhrman nuclear grade, presence of sarcomatoid differentiation, micro-vascular invasion, necrosis, collecting system invasion $(2,30)$.

Clinical factors. The classic triad of flank pain, gross hematuria, and palpable abdominal mass is now rare $(6-10 \%)(9,43)$. The presence or absence of classical symptoms mentioned above, along with constitutional symptoms has shown o have some prognostic significance in local disease. Performance status (PS) assessed by the ECOG or Karnofsky has been found to have significant association with prognosis in several studies. It has been proved by the study at University of Michigan that the way of presentation of the disease symptomatic vs. incidental may also serve as an independent prognostic factor for survival (26).

Molecular Factors. Numerous molecular markers have been studied for the use in the management, prognosis and follow up of local RCC. However, at present the available data doesn't support routine clinical application of any. Panels of several markers are likely to be of higher predictive value than a single one. Further research is needed to assess the utility of biomarkers in the clinical work-up of patients with RCC (11).

\section{PROGNOSTIC FACTORS IN METASTATIC RCC}

Anatomical factors. In the metastatic setting, the classical anatomical factors (stage, size, venous, adrenal invasion) that have been previously described for local RCC, have limited prognostic role. It is generally considered that the prognostic impact of primary tumor variables decreases as soon the tumor spreads and becomes metastatic. Anatomical site of metastases has some prognostic role, but resectability of metastases is even more relevant independent prognosticator regardless of the site. In case of lung metastases, the presence of multiple lesions and associated lymph node involvement correlates with worse outcomes. Patients with greater than one site of metastases, are associated with poorer prognosis (20).

Histological factors. The histological features of possible prognostic value in metastatic RCC are histological subtype and the presence of sarcomatoid component. Taking into account that non-clear cell tumors are worse responders to cytokines, this has been considered an important factor in the era of immunotherapy. At present, when the antiangenic drugs are becoming the first treatment choice, the predictive relevance of histological subtype needs to be clarified. The presence of sarcomatoid differentiation in metastatic RCC is clearly related to worse prognosis (43).

Clinical factors. Clinical picture of the disease becomes of special importance once the tumor develops metastases. There are 4 groups of factors (40):

1) Patient factors include constitutional symptoms such as weight loss, decreased appetite, musculoskeletal pain, sweats, respiratory and gastrointestinal symptoms, having negative impact on survival (40). PS is the most important clinical prognosticator in metastatic RCC. This has been clearly established both in the immunotherapy and targeted therapy era (5). 
2) Tumor burden markers include elevated lactate dehydrogenase (LDH) due to high cell turnover, hypercalcemia due to bone metastases or production of parathyroid hormone related peptide, hyponatremia due to syndrome of inappropriate antidiuretic hormone secretion and anemia $(19,21,14)$.

3) Proinflammatory markers. With the development in cancer research, it has become clear that cancer progression depends on a complex interaction between the tumor and the host inflammatory response. Clinically the systemic inflammatory response can be evaluated by a number of markers: elevated C-reactive protein (CRP) levels, accelerated erythrocyte sedimentation rate (ESR), or increased white cell, neutrophil and platelet counts. Among the inflammatory indicators CRP is reported to be superior to other inflammatory markers. The recent meta-analysis published by $\mathrm{Wu}$ et al. compiling 47 studies' data, suggests that CRP, thrombocyte count and ESR are essential factors for predicting tumor-specific survival with estimated hazard ratios (HR) accordingly $3.46,3.22$ and 3.85 (44).

4) Treatment-related factors include presence or absence of previous nephrectomy and duration of disease free interval (DFI). The interval between nephrectomy and development of metastatic disease is inversely linked to prognosis (7). For patients who present with metastatic disease and good PS cytoreductive nephrectomy prior to imunotherapy improves survival if compared to interferon-a alone, that has been proved by randomized studies (12).

Molecular factors. A large number of genes and proteins have been tested as potential prognostic factors for metastatic RCC. Since targeted therapies are directed to well-defined molecular targets, there is a strong rationale to assess these targets also as prognostic markers. For example, the VEGF gene family, CAIX, VEGFRs, PDGFRs, VHL status are good candidates to analyze the VHL pathway in clear cell RCC, but pAkt, PTEN, p27, and pS6 can be useful for exploring the mTOR pathway in clear cell and non-clear cell RCC. However, no study has clearly established yet the usefulness of one or more of these factors in the routine clinical practice $(17,11)$.

\section{PROGNOSTIC SYSTEMS}

Most of the features mentioned above, in particular TNM stage, Fuhrman nuclear grade and PS can be used as independent prognostic factors. However to achieve higher accuracy in outcome prediction the use of integrated systems that combine multiple independent prognostic variables is recommended. Models proposed by various authors differ by analyzed variables, selected patient population, predicted outcomes and prognostication methods: stratification into risk groups or individual risk assessment. However, not all of them are appropriate for daily clinical use and decision making, because of following drawbacks:

1) do not provide individual level predictions;

2) are weighted towards limited number of patients;

3) no external validation;

4) applicable for selected patient population with definitive histology, stage or previous treatments.
All these criteria have to be considered, when selecting the most appropriate model in the particular clinical scenario, which complicates and limits their routine use outside investigational projects. In this article, we summarized the currently proposed models for prediction of outcomes for both surgical and pharmacological treatments in RCC. The aim of this overview is to encourage the clinicians in using the suggested scoring algorithms and nomograms for decision making in specific clinical situations. Variables used in the models are not detailed as most of them are detected in routine clinical setting. At the time this article is created there at least several comprehensive reviews available $(11,17,26,38,40,43)$ and we will not go into thorough description of each system, but will try to emphasize the most useful ones, by selecting the models of most clinical utility in assisting physicians in their intense work.

The ideal prognostic model should meet the following criteria $(13,15)$ :

1) should be calibrated and externally validated in the independent patient group producing high accuracy;

2) should reflect the situation not just in one center, but in wider population;

3) should meet the contemporary requirements, when new diagnostic and therapeutic techniques are available comparing with the historical patient cohort;

4) should provide individual risk evaluation of each patient;

5) should be simple and easy to use;

6) should not include costly or complicated tests, which cannot to used in clinical practice or are inaccessible on daily basis.

Unfortunately, in general, prognostic accuracy of the models recommended for metastatic disease is significantly worse as for local RCC. One of the possible reasons for that is that information on the primary renal tumor is excluded e.g., histological factors and status of the lymph nodes. It can be partially explained by the lack cytoreductive operations in historical series in metastatic renal tumors. Theoretically, it is possible that inclusion of the primary tumor characteristics could improve the prognostic accuracy; however, today more attention is paid to molecular markers. It is therefore expected that further improvements will be related to inclusion of biological and genetic features in the currently approved systems $(26,38)$.

By reviewing systematic overviews and separate study articles, totally 46 prognostic systems proposed by various authors were identified. When comparing published data, the most established and precise models where selected. To make practical application more convenient the proposed models are grouped according to the purpose they can be used for.

1) Due to the wider availability of imaging techniques, the number of patients with small, asymptomatic renal masses has increased. Excision is the usual standard care in such cases, although only 10$30 \%$ of them are potentially aggressive. Several 
prenephrectomy models are created to predict the presence of RCC, possible metastatic progression and RCC-specific mortality. Over the past decade, at least 9 models predicting different endpoints prior to nephrectomy have been developed (17). We would like to mention here model created by Kutikov et al. that allows selection of patients that are at higher risk of death from RCC as from other causes (23). In low-risk patients close monitoring of the disease or less aggressive ablation treatments could be offered. The nephrometry scale proposed by the same author is another useful tool in evaluating the potential of tumor malignancy prior operation (24).

2) UCLA Integrated Staging System (UISS) and the Mayo Clinic's SSIGN score for many years have been the two most used prognostic models for localized RCC $(45,13)$. Several new predictive systems for estimating survival outcomes after nephrectomy for patients with localized RCC are developed in the past decade. There have been attempts of doing direct comparison between the proposed models, however the obtained data is not convincing in favor to one certain model. Currently there are several ongoing adjuvant treatment trials in highrisk patients after nephrectomy in which different models for risk estimation are selected. More evidence is expected after their completion (15). In 2009 Karakiewicz et al. proposed a new nomogram for prognosis of RCC, in which tumor size is used as a continuous variable and the ECOG performance status is replaced by symptom classification, which is likely to be more acceptable to urologists than PS (18). Another beneficial feature of this model is its abiliy to provide individual estimation of RCCspecific survival instead of grouping patients into risk categories. Furthermore, the multi-institutional data set makes this model more likely to be applicable for patients treated with nephrectomy at other centers. The Karakiewicz model is easy to use (online version is available), has high predictive accuracy, which makes it attractive for individual patient counseling. A recent study by Tan et al. has demonstrated that the postoperative Karakiewicz nomogram achieves superior survival prediction providing higher clinical benefit, comparing to other tested models (39).

3) For prediction of recurrence in local RCC after nephrectomy the system created in 2005 by Sorbellini et al. could be helpful (37). This model achieved $82 \%$ accuracy in external validation, but is applicable only to clear cell RCC.

4) Metastatic RCC has a very poor prognosis with 5year survival not exceeding 20\% (45). However the natural course of the disease in these patients may differ a lot. Several prognostic models have been proposed, but only few have been assessed for their predictive accuracy. The French group of immunotherapy (35) and different versions of Motzer's models $(33,32)$ from the Memorial SloanKettering center (MSKCC) are the two prognostic tools, which have been widely adapted in the clinical practice. 5 prognostic factors that have been identified by multivariate analysis including low Karnofsky PS, elevated LDH, low serum hemoglobin, high corrected serum $\mathrm{Ca}$, absence of nephrectomy or time from diagnosis to initiation of systemic therapy, depending on the version, are used for patient stratification in the Motzer's models. Despite its acceptance by clinicians, it has few drawbacks, including no conclusive predictive accuracy, lack of the variables for the primary tumor and no consideration of lymph node status. A slightly modified version of the Motzer's criteria have been tested by Escudier et al. adding other two variables: alkaline phosphatase (AP) and number of metastatic sites (8). Another model has been proposed by Leibovich et al., along with the prognostic value, the importance of which is related to a subgroup of 192 patients in whom the metastatic RCCs were completely resected, that resulted in approximately $60 \%$ survival at 3 years after surgery (27). This finding emphasizes the need for surgical treatment in feasible patients at least by the time systemic therapies for metastatic RCC do not prove a curative effect.

Two other systems developed recently and being of potential interest are the one published by Iimura et al. (16) with reported external validation score of $86 \%$, and the second one by Manola et al. (29) including data of 3748 patients in clinical trials with separate data set from patients on tyrosine kinase inhibitors. 9 prognostic factors were identified for survival in metastatic disease: PS, number of metastatic sites, time from diagnosis to treatment, pretreatment hemoglobin, white blood cell count, $\mathrm{LDH}, \mathrm{AP}$ and serum calcium.

5) The current selection of targeted agents has been mostly based on clinical efficacy, side effect profile, comorbidities and PS. However, with a rapid expansion of targeted drugs, choosing the most appropriate one is becoming increasingly difficult. That's why recently the significance of prognostic systems in metastatic setting has particularly increased in prediction of the response to systemic treatment. Motzer et al (34). reported the first nomogram to predict 12 months progression-free survival after first-line treatment with sunitinib. Another prognostic system was proposed by Choueiri et al. to determine survival after antiangiogenic therapy (3). A multivariate analysis of risk factors adversely associated with PFS identified an ECOG PS score $\geq 1$, time from diagnosis to treatment $<2$ years, and corrected serum calcium level $>10 \mathrm{mg} / \mathrm{dl}$. Two additional risk factors that have been identified in previous studies, high platelet count $(>300 \mathrm{~K} / \mathrm{mcl})$ and high absolute neutrophil count $(>4.5 \mathrm{~K} / \mathrm{mcl})$, were of some significance as well. Similar model has been applied by Heng et al. by analyzing a cohort of 645 patients with metastatic RCC (all subtypes) treated with VEGF-targeted agents (14). 
At present patient risk stratification proposed by Motzer (33) based on 5 predictive factors as mentioned above is recommended by the guidelines of European Association of Urology (EAU) (28) and ESMO (9) for selection of systemic treatment in metastatic setting and has become an accepted standard in many clinics. According to the National Comprehensive Cancer Network (NCCN) guidelines (31) only poor-prognosis group is distinguished in which temsirolimus is recommended based on the extended 6-factor model provided by Hudes et al., in addition to 5 Motzer's criteria the presence of multiple metastases is also considered (15).

6) For prediction of survival in patients having recurrence after nephrectomy, special model proposed by Eggener et al. based on similar criteria as suggested by R. Motzer, may be used (6).

7) For selection of patients with metastases that could benefit from surgical treatment a special model has been developed by Culp at al. (4). Since the highrisk patients experience significantly lower benefit from cytoreduction, any other algorithm applicable for metastatic RCC can be used for this purpose (26).

8) After surgical excision, $20 \%$ to $30 \%$ of patients with localized tumors experience relapse. The median time to relapse after surgery is 1 to 2 years, with most recurrences occurring within 3 years (28). At present there is no consensus on surveillance after treatment for RCC. The main reason for control is to identify local recurrence or metastases early. Intensive radiological surveillance for all patients is unnecessary. For example, the outcome after surgery for Tla, low-grade, tumors is almost always excellent. It is therefore reasonable to plan followup, taking into account the risk of a recurrence. Scoring systems and nomograms designed by Liebovich, UCLA, and Karakiewicz $(27,45,18)$, can be easily adapted to estimate the likelihood of RCC patients of developing tumor local recurrence or metastases. As recommended by EAU guidelines (28) surveillance after treatment for RCC should be based on a patient's risk group: for low-risk disease, the use of CT can be infrequent; in the intermediate-risk group, an intensified follow-up that includes CT scans at regular time intervals should be performed according to a risk-stratified nomogram; in high-risk patients, the follow-up examinations should include routine CT scans.

According to (NCCN) guidelines (31) no single follow-up plan is appropriate for all patients and it should be individualized based on the patient and tumor characteristics.

Lifelong surveillance is necessary only for some patients with RCC. Late recurrences more than 10 to 20 years after nephrectomy are rare, but single cases have been reported as long as 45 years after initial surgical resection (41). The appropriate intensity of follow up after 5 years remains to be established.
9 Most of the nomograms are designed after 1999 and does not include any of the possible biomarkers. It is expected that the incorporation of molecular factors into standard predictive nomograms may lead to higher prognostic and predictive accuracy. As a result recently several models have been created with integrated molecular information.

Kim et al. screened RCC patients using a tissue microarray technique to examine 29 markers related to the hypoxia-inducible and rapamycin pathways were evaluated (22). A unified nomogram was developed including Ki-67, p53, endothelial VEGFR-1, epithelial VEGFR-1, and epithelial VEGF-D along with ECOG PS, T stage, Fuhrman grade to stratify patients into risk groups. Addition of molecular markers along with classic variables improved the predictive accuracy considerably (concordance index of 0.9). Parker et al. created another biomarker-based scoring system in patients with clear cell RCC called BioScore by integrating information on B7-H1, Ki-67, and survivin. Patients with high BioScores $(>4)$ were 5 times more likely to die from RCC than those with low scores. In addition, the sequential use of BioScore with existing scoring systems (TNM, UISS, SSIGN) enhanced their predictive ability compared to each of these scoring systems alone, which makes it quite attractive for further evaluation in investigational and clinical settings (36). Neither clinical data, nor biological information can be treated in isolation, as both are relevant to patient care and outcomes. It is expected that the future success of biomarker studies will lead to modification of existing systems or developing new ones based on large-scale multivariate analysis.

More experienced professionals when assessing the prognosis and selecting the appropriate treatment in RCC patients use their observations and intuition developed over the years, the youngest colleagues act in accordance to literature studies. However, even if the one is fairly confident in relying just on personal feelings and knowledge, it needs to be considered that more objective methods for prediction and management of this heterogeneous disease are available.

\section{CONCLUSIONS}

1) In several European countries, including Latvia, not only morbidity but also mortality rates of RCC continue to increase.

2) Survival of RCC patients depends mainly on a TNM stage; however, among the patients of each stage different course of disease is observed. Certain factors - analyzed separately and in combinations are essential for prognosis of RCC outcomes and prediction of treatment response.

3) 46 prognostic systems developed by several authors have been identified, having less or more significant differences in the analyzed outcomes and variables, selected patient populations, previous treatments used and purpose of application.

4) The most accurate and commonly used models have been selected according to their intended use, including management of small renal masses, 
identification of patients requiring cytoreductive or systemic treatment, guidance of the follow up and advising on disease outcomes. We propose a simple decision making algorithm in common clinical scenarios throughout entire history of the disease in patients with RCC by indicating the possible prognostic systems to be used at each step (Table 1).

\section{Conflict of interest: None}

\section{REFERENCES}

1. Bedke J, Buse S, Pritsch M, et al. Perinephric and renal sinus fat infiltration in pT3a renal cell carcinoma: possible prognostic differences // BJU International, 2009; 103(10):1349-1354

2. Cheville JC, Lohse CM, Zincke H, et al. Comparisons of outcome and prognostic features among histologic subtypes of renal cell carcinoma // Am J Surg Pathol, 2003; 27:612-624

3. Choueiri TK, Garcia JA, Elson P, et al. Clinical factors associated with outcome in patients with metastatic clear-cell renal cell carcinoma treated with vascular endothelial growth factor-targeted therapy // Cancer, 2007; 110:543-550

4. Culp SH, Tannir NM, Abel EJ, et al. Can we better select patients with metastatic renal cell carcinoma for cytoreductive nephrectomy? // Cancer, 2010; 116:3378-3388

5. de Reijke TM, Bellmunt J, van Poppel H, et al. EORTC-GU group expert opinion on metastatic renal cell cancer // Eur J Cancer, 2009; 45:765-773

6. Eggener SE, Yossepowitch O, Pettus JA, et al. Renal cell carcinoma recurrence after nephrectomy for localized disease: predicting survival from time of recurrence // J Clin Oncol, 2006; 24(19):31013106

7. Elson PJ, Witte RS, Trump DL. Prognostic factors for survival in patients with recurrent or metastatic renal cell carcinoma // Cancer Res, 1988; 48:73107313

8. Escudier B, Choueiri TK, Oudard S, et al. Prognostic factors of metastatic renal cell carcinoma after failure of immunotherapy: new paradigm from a large phase III trial with shark cartilage extract AE 941 // J Urol, 2007; 178:1901-1905

9. Escudier B, Kataja V; ESMO Guidelines Working Group. Renal cell carcinoma: ESMO Clinical Practice Guidelines for diagnosis, treatment and follow-up // Ann Oncol, 2010; 21(5):137-139

10. European Cancer Observatory (ECO) // eco.iarc.fr

11. Finley DS, Pantuck AJ, Belldegrun AS. Tumor biology and prognostic factors in renal cell carcinoma // Oncologist, $2011 ; 16(2): 4-13$

12. Flanigan RC, Salmon SE, Blumenstain BA, et al. Nephrectomy followed by interferon alfa$2 b$ compared with interferon alfa-2b alone for metastatic renal-cell cancer // N Engl J Med, 2001; 345:1655

13. Frank I, Blute ML, Cheville JC, et al. An outcome prediction model for patients with clear cell renal cell carcinoma treated with radical nephrectomy based on tumor stage, size, grade and necrosis: the SSIGN score // J Urol, 2002; 168:2395-2400

14. Heng DY, Xie W, Regan MM, et al. Prognostic factors for overall survival in patients with metastatic renal cell carcinoma treated with vascular endothelial growth factor-targeted agents: results from a large, multicenter study // J Clin Oncol, 2009; 27:5794

15. Hudes G, Carducci M, Tomczk P, et al. Temsirolimus, interferon alfa, or both for advanced renal-cell carcinoma // N Engl J Med, 2007; 356, 2271-2281

16. Iimura Y, Saito K, Fujii Y, et al. Development and external validation of a new outcome prediction model for patients with clear cell renal cell carcinoma treated with nephrectomy based on preoperative serum C-reactive protein and TNM classification: the TNM-C score // J Urol, 2009; 181(3):1004-1012

17. Isbarn H, Karakiewicz PI. Predicting cancer-control outcomes in patients with renal cell carcinoma // Curr Opin Urol, 2009; 19(3):247-257

18. Jeldres C, Karakiewicz PI, Suardi N, et al. Conditional survival predictions after nephrectomy for renal cell carcinoma // J Urol, 2009; 182(6):2607-2612

19. Jeppesen AN, Jensen HK, Donskov F, et al. Hyponatremia as a prognostic and predictive factor in metastatic renal cell carcinoma // $\mathrm{Br} \mathrm{J}$ Cancer, 2010; 102:867

20. Kavolius JP, Mastorakos DP, Pavlovich C, et al. Resection of metastatic renal cell carcinoma // J Clin Oncol, 1998; 16:2261-2266

21. Kim HL, Belldegrun AS, Freitas DG, et al. A Paraneoplastic signs and symptoms of renal cell carcinoma: implications for prognosis // J Urol, 2003; 170:1742-1746

22. Kim HL, Seligson D, Liu X, et al. Using tumor markers to predict the survival of patients with metastatic renal cell carcinoma // J Urol, 2005; 173(5):1496-1501

23. Kutikov A, Egleston BL, et al. Evaluating overall survival and competing risks of death in patients with localized renal cell carcinoma using a comprehensive nomogram // J Clin Oncol, 2010; 28(2):311-317

24. Kutikov A, Smaldone MC, Egleston, et al. Anatomic features of enhancing renal masses predict malignant and high-grade pathology: a preoperative nomogram using the RENAL Nephrometry score // Eur Urol, $2011 ; 60(2): 241-248$

25. Lee CT, Katz J, Fearn PA, et al. Mode of presentation of renal cell carcinoma provides prognostic information // Urol Oncol, 2002; 7:135-140

26. Lee LS, Tan MH. Predictive models for the practical management of renal cell carcinoma// Nat Rev Urol, 2012; 9(2):73-84

27. Leibovich BC, Cheville JC, Lohse CM, et al. A scoring algorithm to predict survival for patients with metastatic clear cell renal cell carcinoma: a stratification tool for prospective clinical trials // J Urol, 2005; 174:1759-1763 
28. Ljungberg B, Cowan NC B, Cowan NC, et al. EAU guidelines on renal cell carcinoma: the 2010 update // Eur Urol, 2010; 58:398-406

29. Manola J, Royston P, Elson P, et al. Prognostic model for survival in patients with metastatic renal cell carcinoma: results from the international kidney cancer working group // Clin Cancer Res, $2011 ; 17(16): 5443-5450$

30. Moch H, Gasser T, Amin MB, et al. Prognostic utility of the recently recommended histologic classification and revised TNM staging system of renal cell carcinoma: a Swiss experience with 588 tumors // Cancer, 2000; 89:604-614

31. Motzer RJ, Agarwal N, Beard C, et al. NCCN clinical practice guidelines in oncology: kidney cancer. Version 2.2012 // J Natl Compr Canc Netw, 2009; 7:618-30

32. Motzer RJ, Bacik J, Mazumdar M. Prognostic factors for survival of patients with stage IV renal cell carcinoma: Memorial Sloan - Kettering Cancer Center experience // Clin Cancer Res, 2004; 10:6302-6303

33. Motzer RJ, Bacik J, Murphy BA, et al. Interferonalfa as a comparative treatment for clinical trials of new therapies against advanced renal cell carcinoma // J Clin Oncol, 2002; 20:289-296

34. Motzer RJ, Bukowski RM, Figlin, et al. Prognostic nomogram for sunitinib in patients with metastatic renal cell carcinoma // Cancer, 2008; 113:15521558

35. Negrier S, Gomez F, Douillard JY, et al. Prognostic factors of response or failure of treatment in patients with metastatic renal carcinomas treated by cytokines: a report from the Groupe Francais d'Immunotherapie // World J Urol, 2005; 23:161165

36. Parker AS, Leibovich BC, Lohse CM, et al. Development and evaluation of BioScore: A biomarker panel to enhance prognostic algorithms for clear cell renal cell carcinoma // Cancer, 2009; 115:2092-2103
37. Sorbellini M, Kattan MW, Snyder M, et al. A postoperative prognostic nomogram predicting recurrence for patients with conventional clear cell renal cell carcinoma // J Urol, 2005; 173:48-5 1

38. Sun M, Shariat SF, Cheng C, et al. Prognostic factors and predictive models in renal cell carcinoma: a contemporary review// Eur Urol, 2011; 60(4):644661

39. Tan MH, Choong CV, Chia KS, et al. The Karakiewicz nomogram is the most useful clinical predictor for survival outcomes in patients with localized renal cell carcinoma // Cancer, 201 1; 117:5314-5324

40. Tang PA, Vickers MM, Heng DY, Heng YC. Clinical and molecular prognostic factors in renal cell carcinoma: what we know so far // Hematol Oncol Clin North Am, 2011; 25(4):871-891

41. Tapper H, Klein H, Rubenstein W, et al. Recurrent renal cell carcinoma after 45 years // Clin Imaging, 1997; $21(4): 273-275$

42. Terrone C, Cracco F, Porpiglia F, et al. Reassessing the current TNM lymph node staging for renal cell carcinoma // Eur Urol, 2006; 49(2):324-31.

43. Volpe A, Patard JJ. Prognostic factors in renal cell carcinoma // World J Urol, 2010; 28(3):319-327

44. Wu Y, Fu X, Xiaoli Z, et al. Prognostic role of systemic inflammatory response in renal cell carcinoma: a systematic review and meta-analysis // J Cancer Res Clin Oncol, 2011; 137(5):887-896

45. Zisman A, Pantuck AJ, Wieder J, et al. Risk group assessment and clinical outcome algorithm to predict the natural history of patients with surgically resected renal cell carcinoma // J Clin Oncol, 2002; 20:4559-4566

\section{Address:}

Ieva Vaivode

Department of Medical Oncology

4 Hipokrata Street,

Riga, Latvia, LV-1079

E-mail: ievavvd@gmail.com 
Table 1. Decision making algorithm for patients with renal cell carcinoma

\section{Initial investigations}

1. H\&P: Concomitant diseases and treatments smoking history, PS (Karnofsky or ECOG) presence of local symptoms (hematuria, flank mass, pain), severety of constitutional symptoms
2. Laboratory evaluation: FBC, urinalysis, biochemistry: including liver function tests, creatinine, LDH, AP, corrected Ca, CRP

3. Radiological investigations:

Abdominal / pelvic CT (with or without contrast depending on renal function):

Cystic mass: Bosniak classification / Solid mass: Nephrometry scale, Kutikov 2011 (24) To be assessed in all: extension to Gerota's fascia \& surrounded tissues; necrosis; extension to veins; status of regional l/n; extension to adrenal gland; distant metastasesIn all - Chest XR; If clinically indicated - chest CT, bone scan, brain MRI.

\section{Clinical staging, risk evaluation and treatment decision}

\begin{tabular}{|c|c|c|c|c|c|}
\hline \multicolumn{2}{|c|}{ Stage } & Possible treatment & \multicolumn{2}{|c|}{ Decision making } & Stratification tools \\
\hline IA & & $\begin{array}{l}\text { Partial nephrectomy or } \\
\text { radical nephrectomy or } \\
\text { thermal ablation or } \\
\text { active surveillance }\end{array}$ & \multirow{2}{*}{\multicolumn{2}{|c|}{$\begin{array}{l}\text { 1) Tumour factors: e.g., } \\
\text { localization } \\
\text { 2) Patient factors: e.g., PS, } \\
\text { concomitant diseases, age } \\
\text { 3) Risk assessment }\end{array}$}} & Kutikov 2010 (23) \\
\hline \multicolumn{2}{|l|}{ IB } & $\begin{array}{l}\text { Partial or } \\
\text { radical nephrectomy }\end{array}$ & & & \\
\hline \multicolumn{2}{|c|}{ II \& III } & Radical nephrectomy & \multicolumn{3}{|c|}{ Adjuvant therapy in clinical trials } \\
\hline \multirow{4}{*}{$\begin{array}{l}\text { IV } \\
\text { R } \\
\text { E } \\
\text { L } \\
\text { A } \\
\text { P } \\
\text { S } \\
\text { E }\end{array}$} & Solitary metastasis & $\begin{array}{l}\text { Nephrectomy } \\
+ \text { metastasectomy }\end{array}$ & \multicolumn{2}{|c|}{ Risk assessment } & \multirow[t]{2}{*}{ Culp 2010 (4) } \\
\hline & $\begin{array}{l}\text { Potentially } \\
\text { resectable primary } \\
\text { with multiple } \\
\text { metastasis }\end{array}$ & $\begin{array}{l}\text { Cytoreductive } \\
\text { nephrectomy prior to } \\
\text { systemic therapy }\end{array}$ & \multicolumn{2}{|c|}{ Risk assessment } & \\
\hline & \multirow[t]{2}{*}{ Unresectable } & \multirow[t]{2}{*}{$\begin{array}{l}\text { Systemic therapy or } \\
\text { palliative care }\end{array}$} & \multicolumn{2}{|c|}{$\begin{array}{l}\text { 1) Histology } \\
\text { 2) Risk assessment }\end{array}$} & Motzer 2002 (33) \\
\hline & & & $\begin{array}{l}\text { Prediction of } \\
\text { response to: }\end{array}$ & $\begin{array}{l}\text { cytokines } \\
\text { sunitinib } \\
\text { sunitinib, } \\
\text { sorafenib, } \\
\text { bevacizumab } \\
\text { temsirolimus }\end{array}$ & $\begin{array}{l}\text { Negrier } 2002(35) \\
\text { Motzer } 2008(34) \\
\text { Heng } 2009(14) \\
\text { Hudes } 2007(15)\end{array}$ \\
\hline
\end{tabular}

III Histological examination of specimen and pathological staging IV Individualized follow up based on risk assessment

\section{Investigations to be done at control visits}

1) H \& P; 2) Laboratory evaluation: $C B C$, urinalysis, biochemistry: including liver function tests, creatinine, LDH, AP, corrected $\mathrm{Ca}, \mathrm{CRP}$; 3) Radiological investigations: chest XR, abdominal US or abdominal/ pelvic CT

\section{Stratification tools}

All: Zisman 2001 (45);

Karakiewicz 2007 (18)

Local: Kattan 2001 (56)

Metastatic: Leibovich 2005 (45)

\begin{tabular}{|l|l|}
\hline Disease outcome & Prognostic tools \\
\hline Prediction of recurrence in local disease & Sorbellini 2005 (37) \\
\hline Survival prognosis in local disease & $\begin{array}{l}\text { Zisman 2002 (45); Frank 2002 (13); } \\
\text { Karakiewicz 2009 (18) }\end{array}$ \\
\hline Survival prognosis in metastatic disease & Iimura 2009 (16); Manola 2011 (29) \\
\hline Predictions of rapid progression (3 months) in metastatic disease & Negrier 2002 (35) \\
\hline Prognosis of survival after recurrence & Eggener 2006 (6) \\
\hline
\end{tabular}

